\title{
Anal function-preserving subtotal intersphincteric resection/ partial external sphincteric resection with hybrid 2-port hand-assisted laparoscopic surgery (Mukai's operation) for very low stage I rectal cancer: A case report
}

\author{
MASAYA MUKAI ${ }^{1}$, YASUTOMO SEKIDO ${ }^{2}$, HIROSHI FUKUMITSU ${ }^{1}$, HIDEKI IZUMI $^{1}$, \\ TATSUHIKO HOSHIKAWA ${ }^{1}$, TAKAYUKI TAJIMA ${ }^{1}$, KOUSUKE TOBITA ${ }^{1}$, \\ SOTARO SADAHIRO $^{3}$, SEIEI YASUDA ${ }^{3}$ and KYOJI OGOSHI ${ }^{3}$ \\ Departments of ${ }^{1}$ Surgery, and ${ }^{2}$ Pathology, Tokai University Hachioji Hospital, Ishikawa-cho, Hachioji, Tokyo 192-0032; \\ ${ }^{3}$ Department of Surgery, Tokai University School of Medicine, Bohseidai, Isehara, Kanagawa 259-1193, Japan
}

Received February 15, 2011; Accepted June 1, 2011

DOI: $10.3892 / \mathrm{ol} .2011 .327$

\begin{abstract}
A 62-year-old male patient underwent endoscopic mucosal resection (EMR). Additional hybrid 2-port handassisted laparoscopic surgery (HALS) (Mukai's operation) was performed for early rectal cancer located at the distal border of the rectum/below the peritoneal reflection $(\mathrm{Rb})$ region $[\mathrm{SM}$ massive invasion/ly+/vertical margin $(\mathrm{VM}) \mathrm{X}$ ] via a small transverse incision, approximately $55 \mathrm{~mm}$ long, at the superior border of the pubic bone. After the pelvic floor muscles were dissected by laparoscopy-assisted manipulation, transanal subtotal intersphincteric resection (ISR) was performed under direct vision, securing a margin of more than $15 \mathrm{~mm}$ distal to the EMR scar. Partial external sphincteric resection (ESR) was also performed to obtain an adequate $\mathrm{VM}$ at the posterior region of the EMR scar. After bowel reconstruction, the layers were sutured transanally and a temporary covering colostomy was created. The resected specimen contained no residual tumor cells without lymph node metastasis. At 3 months after the operation, digital examination revealed good tonus of the anal muscles without stricture. The patient is currently undergoing rehabilitation of his anal sphincter muscles in
\end{abstract}

Correspondence to: Dr Masaya Mukai, Department of Surgery, Tokai University Hachioji Hospital, Ishikawa-cho 1838, Hachioji, Tokyo 192-0032, Japan

E-mail: mukai.masaya@hachioji-hosp.tokai.ac.jp

Abbreviations: LACS, laparoscopy-assisted colorectal surgery; HALS, hand-assisted laparoscopic surgery; EMR, endoscopic mucosal resection; ISR, intersphincteric resection; ESR, external sphincteric resection; VM, vertical margin; $\mathrm{Rb}$, rectum/below the peritoneal reflection

Key words: anal function-preserving operation, hybrid 2-port handassisted laparoscopic surgery (Mukai's operation), intersphincteric resection, endoscopic mucosal resection, lower rectal cancer, stage I preparation for the colostomy closure. In conclusion, subtotal ISR combined with partial ESR may decrease the need to perform Miles' operation for T1/2 stage I rectal cancer located at the distal border of the $\mathrm{Rb}$ region.

\section{Introduction}

In recent years, the use of minimally invasive laparoscopyassisted colorectal surgery (LACS) has become relatively common (1-5). Pure LACS using 5-6 ports with a small incision of 35-45 $\mathrm{mm}$ in length is mainly employed in Japan, and has been adopted for a wide variety of operations, including additional surgery for stage I colorectal cancer, curative resection of stage II/III colorectal cancer and palliative procedures for stage IV cancer (1-5). However, it is significantly more difficult to perform laparoscopic surgery for low rectal cancer than for colon cancer, and the indications and surgical methods vary among institutions.

Unlike Japan, hand-assisted laparoscopic surgery (HALS) and hybrid HALS combining direct vision/manipulation are more commonly performed in the US and Europe (6-8). HALS allows for direct palpation with good tactile sensation, while the left hand can be used to grasp and manipulate large and heavy tumors. In addition, the operating time is shorter since surgery is performed as an extension of standard laparotomy, and it is easier to learn the necessary skills and techniques. Furthermore, the monitor employed during surgery provides magnified images that assist in safe and accurate manipulation at sites, such as the deep pelvic floor, lower bladder and posterior surface of the prostate, locations where visualization is difficult during open surgery and manipulation is usually carried out under nearly blind conditions (6-8).

Hybrid 2-port HALS (Mukai's operation) was introduced in August 2007. Since then, we have operated on 180 patients, including 100 cases of colon cancer and 80 cases of rectal cancer $(9,10)$. Eligible patients have no cardiopulmonary risk factors, tolerate surgery under general anesthesia for 2-3 h and have no evidence of lateral lymph node metastasis or 
infiltration of other organs, such as the urinary bladder and intestines, with no invasion of the pelvic cavity for rectal cancer patients. A small transverse incision of 45-55 $\mathrm{mm}$ long is made at the superior border of the pubis, and hybrid 2-port HALS is performed to approach structures in the pelvic floor by combining non-pneumoperitoneum/laparoscopy-assisted manipulation with surgery under direct vision. The most critical procedures of rectal transection/anastomosis are performed while simultaneously viewing laparoscopic images on the monitor $(9,10)$.

If Miles' operation is performed for stage I [T1(SM)/2(MP) $\mathrm{N} 0$ ] rectal cancer located at the lower edge of the rectum/below the peritoneal reflection $(\mathrm{Rb})$ region, the patient is left with a permanent stoma that creates a significant mental and physical burden, although the 5-year survival rate is over $90 \%(4,5)$. Therefore, in recent years the technique of intersphincteric resection (ISR) via the transanal approach has been promoted for the preservation of anal function in patients with lower rectal cancer (11-14). When extended low anterior resection is required for a tumor at the lower border of the $\mathrm{Rb}$ region, performing LACS intraperitoneally may result in incomplete resection of the anterior (prostatic) section of the internal anal sphincter. Thus, it is often necessary to employ the transanal approach to ensure successful total/subtotal ISR of the entire circumference of the rectum, such as complete ring-shaped circumferential incision (11-14). However, a number of surgeons prefer a curative Miles' operation for stage I rectal cancer located in the middle of the Rb region with T2 (MP) invasion due to concern with regard to the resection margin and because lymph node metastasis occurs in $15-25 \%$ or more of these patients $(4,5)$.

\section{Case report}

Patient. In August 2010, a 62-year-old male presented to his local doctor with bloody stools. The patient was referred to our hospital for the reason that a soft elevated lesion of $\sim 35 \mathrm{~mm}$ in diameter had been found in the lower rectum. The lesion was suspected to be stage I lower rectal cancer.

The patient's past history and laboratory test results were not contributory.

Barium enema examination. Barium enema revealed an elevated polypoid lesion of approximately $35 \mathrm{~mm}$ in diameter at the lower border of the $\mathrm{Rb}$ region of the rectum on the left posterior wall. The lesion had an irregular surface and was mobile (Fig. 1A).

Colonoscopy and endoscopic mucosal resection. Colonoscopy revealed a tumor located just above the dentate line and $\sim 15 \mathrm{~mm}$ proximal to the distal border of the $\mathrm{Rb}$ region on the left posterior wall of the rectum. The tumor had an irregular surface and was hemorrhagic, soft and mobile when light pressure was applied with forceps (Fig. 1B). Endoscopic mucosal resection (EMR) was performed since, i) there was no histological evidence of cancer, ii) there was no risk of perforation and, iii) complete retrieval of the resected tissues was anticipated (15). The tumor was completely resected in two sections and the defect was completely closed by endoscopic clips (Fig. 1C and D). Histopathological exami-

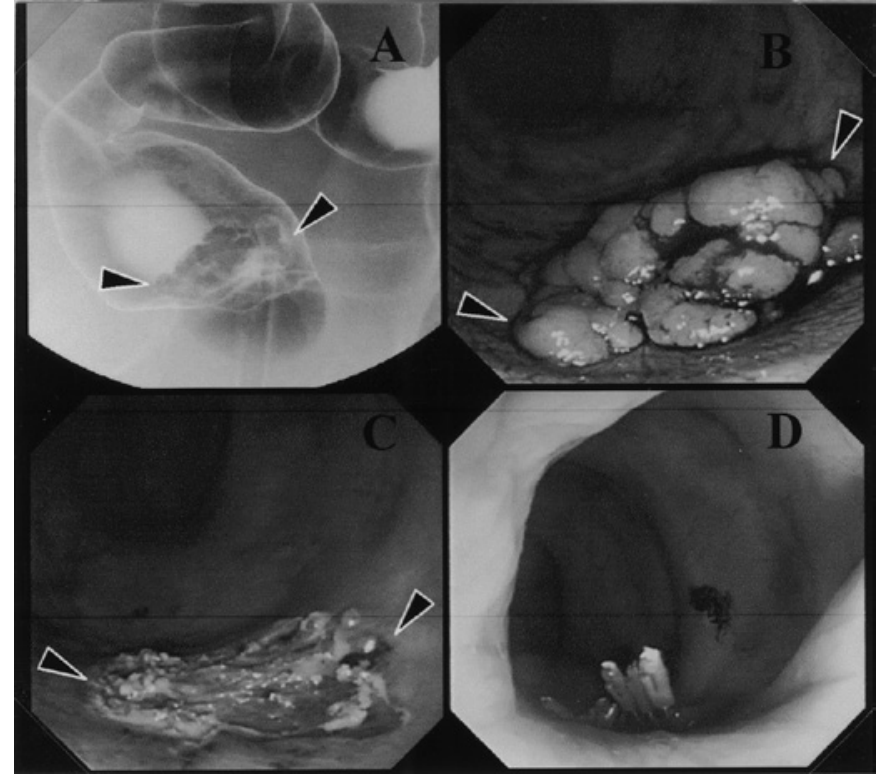

Figure 1. (A) An elevated polypoid lesion of $\sim 35 \mathrm{~mm}$ in diameter was detected at the distal border of the $\mathrm{Rb}$ region of the rectum on the left posterior wall. The lesion had an irregular surface, but was well-demarcated and mobile. (B) A tumor was detected just above the dentate line and $\sim 15 \mathrm{~mm}$ proximal to the distal border of the $\mathrm{Rb}$ region. The tumor had an irregular surface and was hemorrhagic, soft and mobile when light pressure was applied with forceps. (C and D) The tumor was completely resected in two sections and the defect was completely closed with endoscopic clips.

nation revealed a well-differentiated adenocarcinoma with massive submucosal (SM) invasion $(\geq 1,800 \mu \mathrm{m})$ and ly1/v0. The vertical margin (VM) could not be determined (VMX).

Based on these findings, the patient was diagnosed as having stage I lower rectal cancer (pSM massive invasion/ $\mathrm{ly}(+), \mathrm{pVMX})$ and additional curative resection combined with lymph node dissection was performed $(4,5)$.

Additional operation (hybrid 2-port HALS; Mukai's operation with a transanal procedure). A small transverse incision of $\sim 55 \mathrm{~mm}$ long was made above the superior border of the pubic bone, and hybrid 2-port HALS was performed deep in the pelvic floor by combining non-pneumoperitoneum/ laparoscopy-assisted manipulation with procedures under direct vision. Dissection of the puborectal, rectococcygeal and pelvic floor muscles was performed by laparoscopy-assisted manipulation $(9,10)$. Following dissection of the area proximal to the dentate line, the tumor resection scar was identified under direct vision via the transanal route. With a margin of $>15 \mathrm{~mm}$ distal to the tumor resection scar, complete circumferential resection and transanal subtotal ISR were performed (11-14). Since VM was not determined pre-operatively, partial external sphincteric resection (ESR) was also performed, with the rectum being resected one layer deeper in order to obtain an adequate margin (16-19) (Fig. 2A and B). For reconstruction, the colon was pulled through to the external sphincter muscle cone at the dentate line (Fig. 3A). There was no tension with good color at the anastomotic site. The layers were sutured by hand sewing with Albert's interrupted sutures (Fig. 3B). In addition, a temporary covering colostomy was created with the wound closure of a small transverse incision (Fig. 4A and B). 


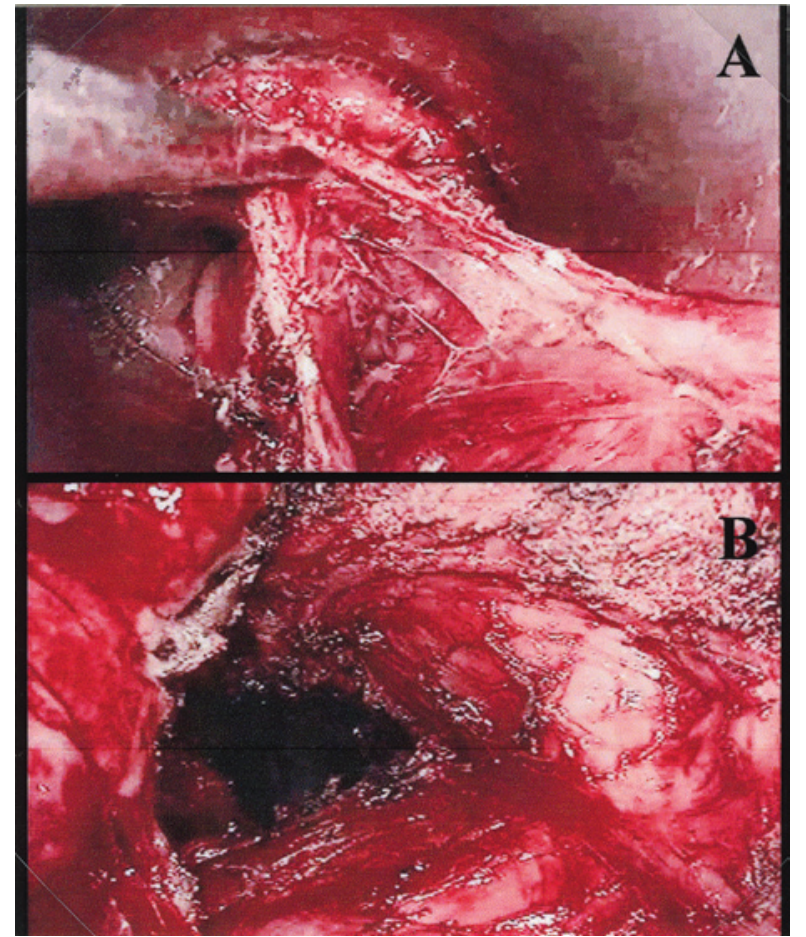

Figure 2. Transanal subtotal internal sphincter resection (ISR) was performed with a margin of $>15 \mathrm{~mm}$ at the distal side of the tumor resection scar. On the posterior side, partial external sphincter resection (ESR) was performed, with the rectum being resected one layer deeper in order to obtain an adequate margin.

\section{Results}

Pathological diagnosis. On histopathological examination, no residual cancer cells were detected at the EMR site or elsewhere in the resected specimen [stage I pN0 (0/12) and pAW(-)] (Fig. 5A-C). The layers of the internal anal sphincter were completely resected (Fig. 5A and B), and the external anal sphincter with striated muscle fibers was detected at the resection margin (VM-) posterior to the EMR site (Fig. 5B and D).

Postoperative course. The patient had a favourable course and was discharged on the 13th postoperative day. Three months after the operation, digital examination revealed good tonus of the anal muscles. The patient is currently undergoing anal sphincter rehabilitation to prepare for the colostomy closure.

\section{Discussion}

A number of institutes in Japan now perform transanal procedures for lower rectal cancer, particularly the rectal prolapsing/ reversion technique for patients with small tumors up to stage I $(20,21)$. The rectal prolapsing technique allows safe and adequate transection of the rectum under direct vision, but it is occasionally difficult to pull the rectum through to the anus as reversal of the rectal mucosa is hindered by the thickness of the mesocolon. Moreover, ESR cannot be performed. ISR is usually performed transanally as it requires complete circumferential resection of the rectum.

Anatomically, fibers of the longitudinal combined muscles that traverse the internal anal sphincter pass directly below the

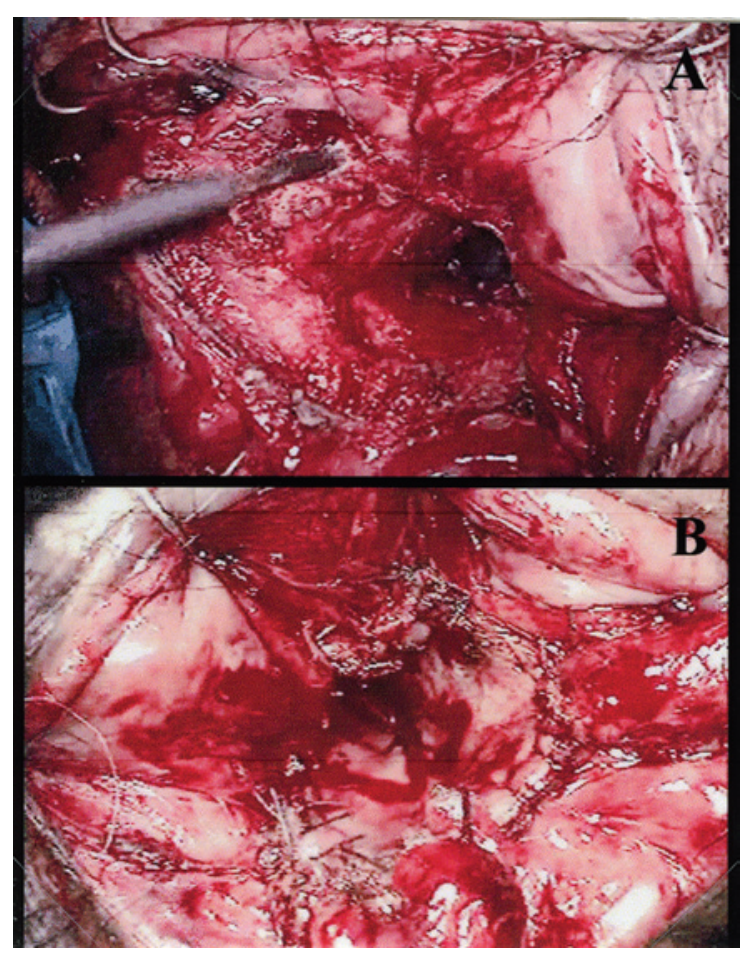

Figure 3. (A) For reconstruction, the colon was pulled through and attached to the external sphincter muscle cone around the dentate line. (B) There was no tension at the anastomosis and the tissues showed good color. The layers were sutured by hand sewing with Albert's interrupted sutures.

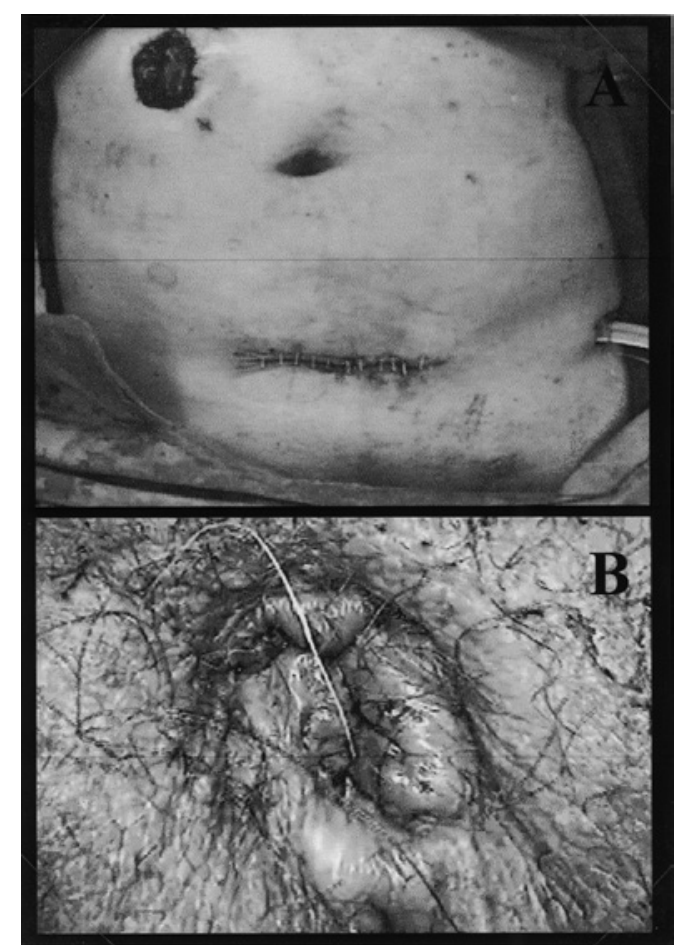

Figure 4. (A) A small transverse incision of $\sim 55 \mathrm{~mm}$ long was made above the superior border of the pubis, and hybrid 2-port HALS (Mukai's operation) was performed with a temporary covering colostomy and (B) the postoperative view of the anus.

anal verge. Therefore, it may be considered more appropriate from a histological point of view to use the term 'subtotal ISR' when dissection is performed below or proximal to the dentate 


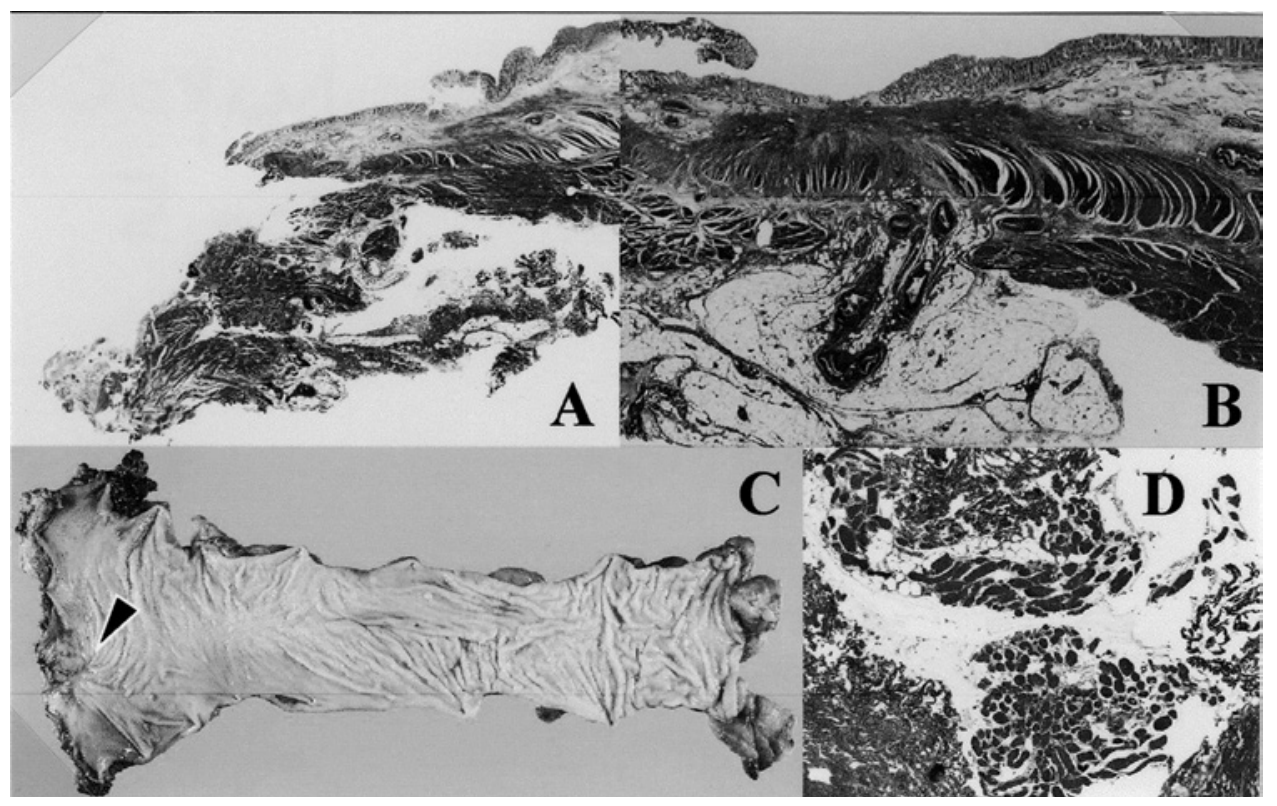

Figure 5. (A and B) On histological examination, all layers of the internal anal sphincter were completely resected. (B and C) No residual cancer cells were detected at the EMR site or elsewhere in the resected specimen [pN0 (0/12) and pAW(-)]. (B and D) The external anal sphincter with striated muscle fibers was identified at the resection margin (VM-) posterior to the EMR site.

line, as opposed to 'total ISR', which describes subcutaneous dissection up to the distal edge of the anal epithelium (11-14). In addition, ISR performed intraperitoneally may also be regarded as partial ISR since the incision is arc-shaped at the anterior (prostatic) side of the rectal wall and ring-shaped circumferential rectal resection is not achieved. Based on the original report by Parks et al, ISR is defined as complete circumferential rectal resection performed via the transanal approach $(11,12)$.

In the present patient, hybrid 2-port HALS was employed, which allowed laparoscopy-assisted dissection of the internal anal sphincter from the puborectal, rectococcygeal and pelvic floor muscles. This procedure was followed by transanal subtotal ISR, in which circumferential rectal resection was performed with a margin of approximately $15 \mathrm{~mm}$ distal to the EMR scar, which was located proximal to the dentate line under direct vision. Since VM had not been determined pre-operatively to avoid incomplete resection, arc-shaped partial ESR was performed one layer deeper posterior to the EMR site to obtain an adequate resection margin and to ensure complete removal even if the depth of tumor penetration was found to be T1 (SM massive invasion $\geq 1,000 \mu \mathrm{m}$ or $2 / 3$ deep layers) or T2 (minimal MP invasion, such as $1 / 3$ superficial layers or $\leq 1,000 \mu \mathrm{m})$. Although the anal function of this patient may become an issue in the future after stoma closure, postoperative digital rectal examination revealed a favorable tonus of the anal muscles. The patient is currently undergoing daily rehabilitation to increase the strength of his anal sphincter muscles.

Another important point of this surgical technique is reconstruction of the bowel. The length, tension, thickness and volume of the mesocolon are critical issues since the colon has to be pulled through the external anal sphincter cone. In most cases, however, the procedure should pose no problem if the splenic flexure is completely mobilized and a relatively long region of the colon/mesocolon is preserved. In addition, if large blood vessels of the mesocolon are ligated, the colon can be extended sufficiently to cover the distance to the anal anastomotic site. Of note, however, is that sufficient attention should be paid to tissue color and tension at the anastomosis to avoid inadequate blood flow at the margin of the colon, so that ligation of the vessels during the operation requires careful judgment. Although construction of a covering loop stoma may theoretically be avoided with a transanal anastomotic procedure, we created a colostomy in this patient due to nonsterile operative procedures in the ano-rectal tube and the need for early rehabilitation. There is considerable merit in having a covering loop colostomy if a patient develops a stubborn anastomotic stricture after surgery, since finger bougie dilation of the anastomotic site can be safely performed. However, whether or not a covering colostomy/ileostomy is required for transanal anastomosis remains a subject for future investigation, depending on the type of surgery performed, and requires the accumulation of a large number of cases. Minimally invasive LACS has become more common, and the magnified view provided by the monitor enables safe and accurate manipulation at sites such as the deep pelvic floor, lower bladder, posterior surface of the prostate and anal region, where visualization is difficult to achieve during open surgery and manipulation is usually carried out under nearly blind conditions.

In conclusion, adoption of the subtotal ISR combined with partial ESR is likely to decrease the number of Miles' operations performed in patients with stage I [T1(SM)/2(MP)N0] rectal cancer located at the distal border of the $\mathrm{Rb}$ region.

\section{Acknowledgements}

This study was supported by grants from the Occult Neoplastic Cells Research and Study Group (No. 2010-5007; Tokai University Hachioji Hospital, Tokyo, Japan) and the 
Research and Study Program of Tokai University Educational System General Research Organization (No. 2007-04; Tokai University School of Medicine, Kanagawa, Japan).

\section{References}

1. Jacobs M, Verdja JC and Goldstein HS: Minimally invasive colon resection (laparoscopic colectomy). Surg Laparosc Endosc 1: 144-150, 1991

2. Mukai M, Tokunaga N, Ishida H, Makuuchi $H$, Tajima $T$ and Mitomi T: Clinical experiences with laparoscopic colectomy. Dig Endosc 9: 11-15, 1997.

3. The Clinical Outcomes of Surgical Therapy Study Group: A comparison of laparoscopically assisted and open colectomy for colon cancer. N Engl J Med 350: 2050-2059, 2004.

4. Makuuchi M and Sugihara K: Knacks and Pit Falls: Surgery of the Colon, Rectum and Anus. 2nd edition. Bunkoudou, Co., Ltd., Tokyo, 2004

5. Guidelines 2010 for the Treatment of Colorectal Cancer in Japan. Japanese Society for Cancer of the Colon and Rectum, Tokyo, Japan, 2010

6. Romanelli JR, Kelly JJ and Litwin DE: Hand-assisted laparoscopic surgery in United States: an overview. Semin Laparosc Surg 8: 96-103, 2001.

7. Nakajima K, Lee SW, Cocilovo C, Foglia C, Kim K, Sonoda T and Milson JW: Hand-assisted laparoscopic colorectal surgery using GelPort. Surg Endosc 18: 102-105, 2004.

8. Nakajima K, Lee SW, Cocilovo C, Foglia C, Sonoda T and Milson JW: Laparoscopic total colectomy: hand-assisted vs. standard technique. Surg Endosc 18: 582-586, 2004.

9. Mukai M, Kishima K, Tajima T, Hoshikawa T, Yazawa N, Fukumitsu H, Okada K, Ogoshi K and Makuuchi K: Efficacy of Hybrid 2-port hand-assisted laparoscopic surgery (Mukai's operation) in patients with primary colorectal cancer. Oncol Rep 22: 893-899, 2009.

10. Mukai M, Hoshikawa T, Fukumitsu H, Yazawa N, Okada K, Tajima T, Sekido Y, Nakamura M and Ogoshi K: Two-stage treatment (Mukai's method) with hybrid 2-port HALS (Mukai's operation) for complete bowel obstruction by left colon cancer or rectal cancer. Oncol Rep 24: 25-30, 2010.
11. Parks AG: Transanal technique in low rectal anastomosis. Proc R Soc Med 65: 975-976, 1972.

12. Parks AG and Percy JP: Resection and sutured coloanal anastomosis for rectal carcinoma. Br J Surg 69: 301-304, 1982.

13. Ito M, Ono M, Sugito M, Kawashima K and Saito N: Curative operation with intersphincteric resection for very low rectal cancer. Dig Surg (In Japanese) 25: 1-11, 2002.

14. Saito N, Moriya Y, Shirouzu K, Maeda K, Mochizuki H, Koda K, Hirai T, Sugito M, Ito $M$ and Kobayashi A: Intersphincteric resection in patients with very low rectal cancer: a review of the Japanese experience. Dis Colon Rectum 49 (Suppl 10): 13-22, 2006.

15. Mukai M, Okamoto Y, Oida Y, Mukoyama S, Ito I, Nakasaki H, Kawai K, Sato S and Makuuchi H: Endoscopic mucosal resection for superficially spreading colonic neoplasms larger than $5 \mathrm{~cm}$ in the right colon after dilute sodium hyaluronate injection: report of two cases. Endoscopy 35: 973-975, 2003.

16. Shirouzu K, Ogata Y, Araki Y, Kishimoto Y and Sato Y: A new ultimate anus-preserving operation for extremely low rectal cancer and for anal canal cancer. Tech Coloproctol 7: 203-206, 2003.

17. Mori S, Ishibashi I, Akagi Y, Ogata Y and Shirouzu K: External sphincteric resection (ESR) as anal function preserving operation for lower rectal cancer: the theoretical grounds. Clin Surg (In Japanese) 64: 339-343, 2009.

18. Akagi Y, Shirouzu K and Kinugasa T: Anal function preserving sphincteric resection: intersphincteric resection (ISR) and external sphincteric resction (ESR). Surgery (In Japanese) 71: 157-162, 2009.

19. Nishikawa S, Aoki K, Yoshikawa T, Tokura $\mathrm{T}$ and Morita $\mathrm{T}$ : Indication and procedure of intersphincteric resection for very lower rectal and anal cancer. Surg Treat (In Japanese) 102: 790-796, 2010

20. Fukunaga M, Kidokoro A, Iba T, Sugiyama K, Fukunaga T, Nagakari K, Suda M and Yoshikawa S: Laparoscopy-assisted low anterior resection with a prolapsing technique for low rectal cancer. Surg Today 35: 598-602, 2005.

21. Idani H, Ishii $\mathrm{T}$, Maruyama $\mathrm{M}$, Ishikawa $\mathrm{T}$, Iwamoto $\mathrm{T}$ and Watanabe S: Laparoscopic low anterior resection using a prolapsing technique for low rectal cancer. Surgery (In Japanese) 67: 1359-1362, 2005. 